\title{
Former Soviet Union / West Europe Consortium for AGN Monitoring
}

\author{
N.G. Bochkarev \\ Sternberg Astronomical Institute, University of Moscow, Universitetskij \\ Prospect 13, Moscow 119899, Russia \\ A.I. Shapovalova and A.N. Burenkov \\ Special Astrophysical Observatory of the Russian AS, Nizhnij Arkhyz, \\ Karachaevo-Cherkesia, 369167, Russia
}

In the same year as Blandford and McKee (1982), Prof. N. Bochkarev and Dr. I. Antokhin (Bochkarev and Antokhin, 1982) published an article on the reverberation method of AGN BLR structure analysis. In 1983, testing observations were carried out with the $6 \mathrm{~m}$ telescope.

From 1986 up to now spectral monitoring of several AGNs has been carried out at SAO RAS (North Caucasus). Since 1988 we have been participating in AGN Watch Program. Since 1997 we have been carrying on an international program that is realised at several observatories of CIS (former Soviet Union) in collaboration with several West-European scientific groups (Bochkarev and Shapovalova, 1999). Some spectra of 3C390.3, one of the dimmest target objects of our program, $m=15.6$ mag,taken with different telescopes are presented in Fig. 1(left).

It should be noted (see Fig. 1) that the $1 \mathrm{~m}$ telescope is too small for obtaining spectra of good quality $(\mathrm{S} / \mathrm{N} \approx 30)$, unless long exposure times are used. Therefore the most usable spectra should be taken with telescopes $\geq 2 \mathrm{~m}$.

In Fig. 1 (right) the light curves for the emission $\mathrm{H} \beta$ line and the continuum of 3C 390.3 obtained in 1995-2000 are shown. The BVRI broad-band monitoring was carried out at 4 CIS observatories with small telescopes $(60 \mathrm{~cm}$ and $70 \mathrm{~cm})$. The errors of the photometric data are about 0.01-0.03 mag. From Fig. 1 (right) we see that the monitoring using photometric data (for continuum) and spectral data (for continuum and $\mathrm{H} \beta$ emission line) agrees each other well.

Presently our consortium on AGN monitoring includes 12 teams from 9 countries (about 50 participants, 19 of them younger than 35 ). We have observational bases located at different hour zones, and teams with vast experience in observations of AGN: optical broad-band photometry - at 5 CIS observatories (three other observatories, including one situated in Siberia, one in the Central Asia in a place with very good astroclimate, and one of the Trans-Caucasus observatories, are ready to join the program); CCD-spectroscopy - at 4 observatories (one of them situated in Mexico); near-IR-photometry - at one observatory; 4 groups involved in X-ray observations (mainly in Western Europe); 5 groups of interpretators, including 4 using photoionization codes CLOUDY and others, as well as groups of theoretical researches.

Besides, our consortium possesses unique archival data: 10-30 year long dense time series of observations of many objects: photoelectric UBV-photometry 

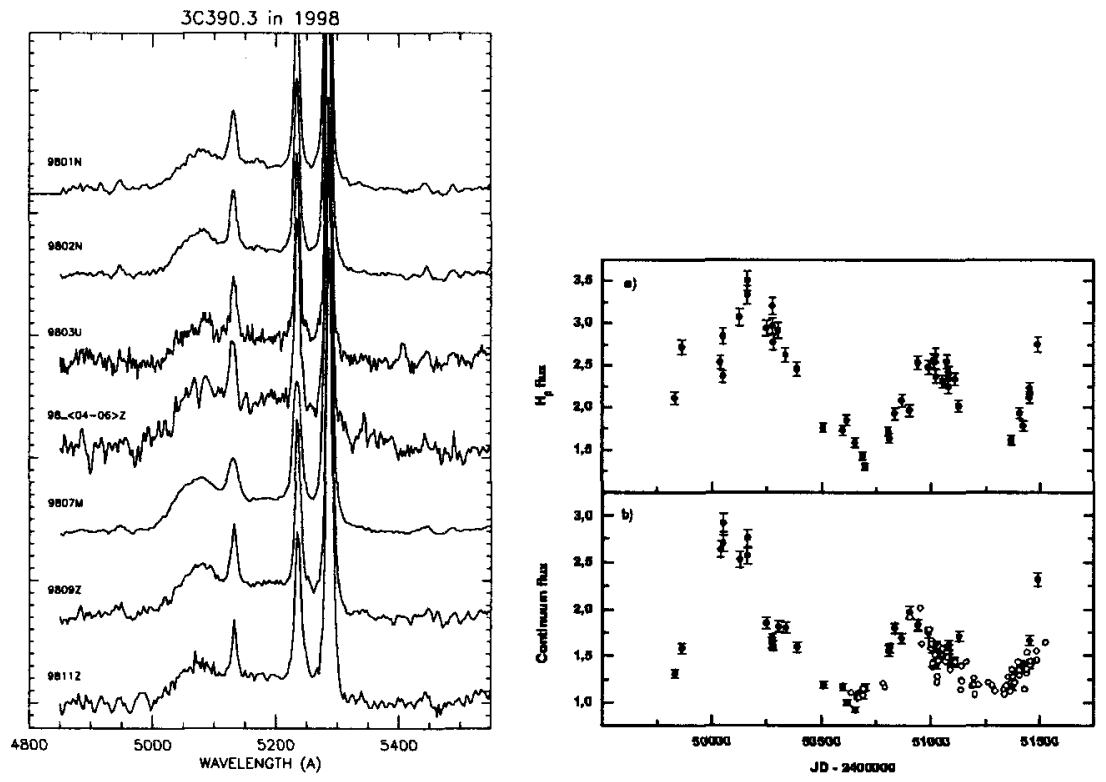

Figure 1. Left: The spectra of $3 \mathrm{C} 390.3$ in $\mathrm{H} \beta$ region observed in 1998 , each labeled with year (98), month $(01=\mathrm{Jan}, 02=$ Feb etc.), and telescope ( $\mathrm{N}=6 \mathrm{~m}$ telescope Nasmyth focus; $\mathrm{U}=$ Prime focus; $\mathrm{Z}=1 \mathrm{~m}$ SAO telescope; $M=2.1-\mathrm{m}$ GHO telescope (Mex́ico)). From top (9801N) to bottom (9811Z) exposure times in minutes are $30,10,15,60,120$, 540,270 and $\mathrm{S} / \mathrm{N}$ are $43,32,13,11,56,34,34$.

Right: Light curves for the emission $\mathrm{H} \beta$ line and the optical continuum of 3C 390.3 from 1995-2000. Filled circles show the spectral data. Those calculated out of V-band photometry are represented by open circles. Units are in $10^{-13} \mathrm{ergs} \mathrm{cm}^{-2} \mathrm{~s}^{-1}$ for $\mathrm{H} \beta$ fluxes, and $10^{-15}$ ergs $\mathrm{cm}^{-2} \mathrm{~s}^{-1} \mathrm{~A}^{-1}$ for the continuum.

with an accuracy of $1 \%$ and low $S / N$ spectra of many AGN since the beginning of 1970 up to now obtained at the Crimean Observatory, Kazakhstan and SAO (Caucasus). Most of the archival spectra remain unprocessed.

This paper has had financial support from INTAS (grant N96-0328), RFBR (grants: N94-02-4885; N97-02-17625; N00-02-16272a), scientific-technical programme "Astronomy" (Russia), RFBR+CHINA (grant 99-02-39120) and CONACYT grants GXXXX-E and 32106-E (México).

\section{References}

Blandford, R.D., McKee, C.F. 1982, ApJ, 255, 419

Bochkarev, N. G., Antokhin, I. 1982, Astron. Tsirk., 1238

Bochkarev N.G., Shapovalova A.I. 1999, 75 (ASPConf.Ser.175) 